\title{
Hématome épidural dorsal spontané
}

\section{Spontaneous Dorsal Epidural Hematoma}

\section{E. Hansconrad}

Reçu le 29 juin 2015 ; accepté le 16 juillet 2015

(C) SFMU et Lavoisier SAS 2015

Un homme de 39 ans sans antécédent particulier est amené aux urgences pour sensation de paresthésies dans les deux membres inférieurs depuis le réveil qui se complique rapidement d'une paraplégie. Il existe également une douleur thoracique à type de brûlure évoluant depuis dix jours, plus intense depuis le réveil. Il n'y a pas eu de traumatisme du rachis récent. Le patient était conscient et orienté, score de Glasgow à 15 . L'examen clinique retrouvait une paraplégie complète avec anesthésie complète sous le niveau T4, un syndrome pyramidal bilatéral avec Babinski bilatéral, une hyperréflexie aux membres inférieurs, des clonies des pieds. Le reste de l'examen clinique était sans particularités. Une IRM médullaire a été réalisée en urgences devant la suspicion de compression médullaire (Fig. 1). Le patient a bénéficié d'une laminectomie T2-T3 avec évacuation de l'hématome extradural. Une artériographie pratiquée en postopératoire était normale. Le patient récupérait progressivement la motricité et la sensibilité en postopératoire. Un hématome épidural spontané est une pathologie extrêmement rare et survient habituellement chez des patients sous anticoagulants ou atteints d'un trouble de la coagulation. Ce diagnostic doit être suspecté devant tout syndrome médullaire associant douleur rachidienne et déficit sensitif et/ou moteur. Une imagerie de la moelle épinière doit être réalisée en urgence afin d'affirmer le diagnostic et permettre au patient de bénéficier d'une chirurgie de décompression [1]. L'IRM médullaire est l'examen de référence permettant d'affirmer la présence d'un hématome épidural, sa localisation et les signes de souffrance médullaire associés. Mais son manque de disponibilité amènera le plus souvent le clinicien à réaliser une TDM médullaire qui met en évidence un hématome épidural spinal sous la forme d'une hyperdensité spontanée comprimant la moelle épinière. La TDM médullaire, bien que moins performante que l'IRM, permet le diagnostic des principales compressions médullaires aiguës : hématome épidural spinal, hernie discale,

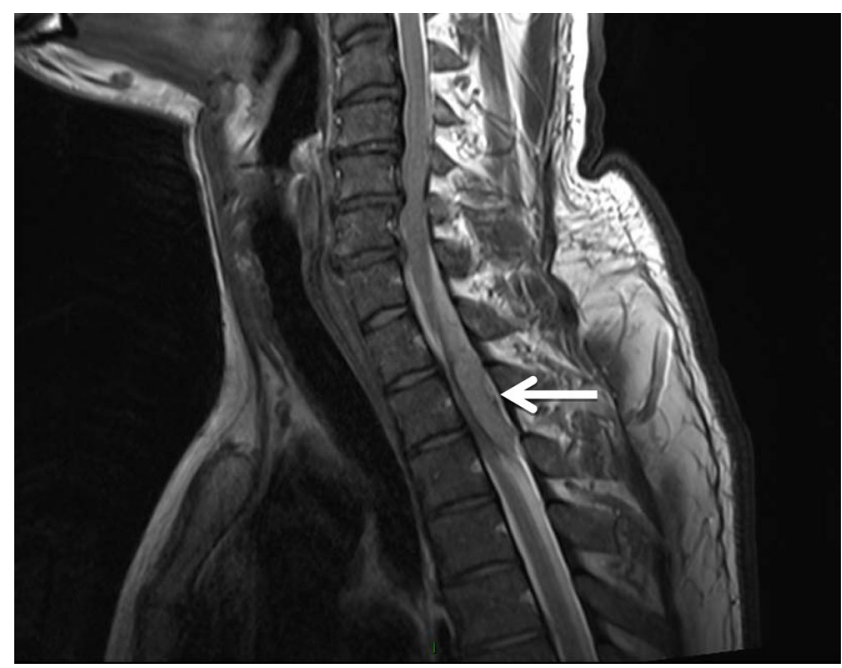

Fig. 1 IRM médullaire en coupe longitudinale, séquence T2, montrant un hématome épidural dorsal récent de $\mathrm{C} 7$ à $\mathrm{T} 3$

tassement vertébral et épidurite infectieuse. En l'absence d'IRM disponible, sa réalisation en urgence est indispensable puisque le pronostic fonctionnel du patient est lié à la précocité du diagnostic et de la prise en charge chirurgicale. L'absence d'étiologie retrouvée sur la TDM médullaire en présence de signes cliniques de compression médullaire nécessite un avis spécialisé neurochirurgical et un complément d'imagerie par IRM médullaire.

\section{Références}

1. Dziedzic T, Kunert P, Krych P, Marchel A (2015) Management and neurological outcome of spontaneous spinal epidural hematoma. J Clin Neurosci 22:726-9
E. Hansconrad $(\bowtie)$

Hôpital Lariboisière, APHP, 2 rue Ambroise Paré, F-75010 Paris e-mail : erwin.hansconrad@hotmail.fr 